\title{
Una mirada crítica del informe de Colombia al Comité sobre los Derechos de las Personas con Discapacidad
}

\section{A critical look of the report of Colombia at the Committee on the Rights of People with Disabilities}

\section{Palabras clave}

Enfoque de capacidades, Martha Nussbaum, Reporte de Colombia, Comité sobre los Derechos de las Personas con Discapacidad.

\section{Keywords}

Capacity approach, Martha Nussbaum, Colombian report, Committee on the Rights of People with Disabilities.
Con el fin de favorecer el desarrollo de las personas con discapacidad, en el año 2006 se establece la Convención sobre los Derechos de las Personas con Discapacidad. En 2013 el Estado colombiano presenta su informe inicial y luego la Sociedad Civil participa mediante un informe alterno (organizaciones, redes de personas con discapacidad y familias, reunidas desde el 20I4 en la "Coalición Colombiana por la Implementación de los Derechos de las Personas con Discapacidad"); en 2016 el Comité sobre los Derechos de las Personas con Discapacidad da a conocer las observaciones finales y recomendaciones sobre el informe presentado por el Estado. Con relación a lo anterior, se realiza un análisis de los artículos I3, I4 I 5 y 2 I a la luz del enfoque de capacidades de Martha Nussbaum (Rodríguez, s.f.).

El Estado colombiano en su Informe al Comité sobre los Derechos de las Personas con Discapacidad señaló, en relación al artículo I 3 (Acceso a la Justicia), que a la luz de la Ley I6I 8 de 20I3, deben inaplicarse las disposiciones previstas en los códigos procesales, normas anteriores a ésta, a criterio de las y los operadores judiciales, quienes deben "realizar los interrogatorios y valorarlos de acuerdo con las reglas de la sana crítica, sin que, por el momento,

\author{
Gina Cabeza Monroy \\ <gcabezam@unal.edu.co> \\ Universidad de Cartagena
}

Natalia Lancheros Martínez

$<$ klancherosm@unal.edu.co>

Universidad del Rosario

\section{Catalina Rodríguez Quintero} <carodriguezqu@unal.edu.co>

Escuela Colombiana de Rehabilitación

\section{Daniel Antonio Sastoque \\ Coronado}

<dasastoquec@unal.edu.co>

Universidad Nacional de Colombia

Para citar:

Cabeza, G. et al. (20I7): "Una mirada crítica del informe de Colombia al Comité sobre los Derechos de las Personas con Discapacidad". Revista Española de Discapacidad, 5 (2): 22I-226.

Doi: <https://doi.org/IO.5569/23405 I04.05.02.I3> 
sea necesaria la expedición de una nueva normativa” (República de Colombia, 2013).

En el Informe alternativo de la Coalición colombiana para la implementación de la Convención sobre los Derechos de las Personas con Discapacidad (Comité sobre los Derechos de las Personas con Discapacidad, 2016a) se destaca la inconstitucionalidad (por vía del Bloque de Constitucionalidad) del Código General del Proceso, pero no se pronuncia sobre la postura del Estado.

Las Observaciones finales sobre el informe inicial de Colombia del Comité sobre los Derechos de las Personas con Discapacidad (20I6b) sugieren el retiro del ordenamiento jurídico del artículo 2 Io del Código General del Proceso.

Jurídicamente la respuesta del Estado colombiano es aceptable, porque se trata de una norma anterior a la ley "Por medio de la cual se establecen las disposiciones para garantizar el pleno ejercicio de los derechos de las personas con discapacidad", luego lo que se evidencia es que en el caso colombiano existe una seria falla en los procesos de formación básica del personal de la rama judicial (pues ello no se limita sólo a juezas y jueces, sino que también aplica a sustanciadoras y sustanciadores y demás personal que funge como tal), en el cual hay que ver la responsabilidad que le asiste a las facultades de derecho y a las escuelas de formación de la rama judicial y la Fiscalía General de la Nación. Aunado a lo anterior, se evidencia una grave falla en la prestación del servicio legislativo pues el Congreso de la República aprobó tal disposición en clara contravía de la Convención que forma parte del Bloque de Constitucionalidad. Lo mismo se predica del Gobierno Nacional que la sancionó.

Esta opción, sin embargo, no es eficaz, pues como lo recuerda Aimee Mullins, "el lenguaje crea realidades" y suele ser perverso y violador de los derechos humanos (Mullins, 2009). Un país tan burócrata en el que prevalece la lectura stricto sensu de la norma, en vez de los principios y el bloque de constitucionalidad propiamente dicho solo puede terminar violando la Convención en el día a día de la prestación de servicios del sector Justicia. El informe sombra se queda corto en ello, pues el acceso a la justicia como derecho fundamental de las personas con diversidad funcional no se agota en una función procesal sino en todo el andamiaje social que lo sustenta y que, por vía de la reproducción de un currículum oculto negativo, perpetúan los prejuicios y estereotipos que vulneran a las personas con discapacidad.

Valdría la pena preguntarnos, ¿qué han hecho la Procuraduría General de la Nación y la Defensoría del Pueblo, como representantes de la sociedad y en defensa de los derechos humanos de las personas con diversidad funcional y del interés general para demandar la constitucionalidad de esta norma? ¿Qué ha hecho la administración judicial, las escuelas de la rama judicial y la fiscalía para que la obligación internacional, derivada de la Convención e incorporada en el ordenamiento interno en tanto bloque de constitucionalidad, se cumpla cabalmente?

Desde la mirada de Mullins y de Nussbaum, un enfoque en capacidades supone considerar a las personas con diversidad funcional en toda su dimensión humana y en la lucha contra el delito, la participación de éstas personas también contribuye al cumplimiento de los fines del Estado y la construcción de ciudadanía, por lo cual es de pleno recibo el llamado del Comité a que el Estado colombiano: "Asegure la accesibilidad de instalaciones físicas, materiales, información y comunicación en todo el sistema de justicia, incluyendo la disponibilidad de guías intérpretes e intérpretes de lengua de señas, material en Braille, de lectura fácil y en formatos electrónicos".

Incluso ello supone unas mejores condiciones de superación de la impunidad en la violencia ejercida contra mujeres y niñas con diversidad funcional, quienes a pesar de estar expuestas en forma mucho más dramática a la violencia sexual que el promedio, estas disposiciones les impiden jugar un rol protagónico en la protección de sus derechos, pues ni siquiera 
como víctimas su voz es "legalmente escuchada".

El Estado colombiano en su informe señala que la Fiscalía General de la Nación está optimizando los resultados en los casos de violencia contra sujetos de especial protección constitucional, como las "personas con discapacidad" y que está atendiendo "con enfoque diferencial" a tales usuarios en tanto víctimas, testigos o vinculados a las investigaciones, pero no indica qué proporción de esa población es la destinataria de la Convención para ambas estrategias, ni brinda información detallada por sub-grupos poblacionales o interseccionalidad, tampoco detalla los mecanismos o procedimientos para evaluar su pertinencia, como un protocolo o procedimiento, talleres de sensibilización y capacitación, contratación de profesionales y/o equipos especializados de soporte y apoyo, etc.

También hay que considerar que el ámbito penal es sólo una de las dimensiones de la prestación del servicio público de administración de justicia y es justamente en otras áreas donde más se exponen las personas con diversidad funcional al desconocimiento de sus derechos, como en las áreas civil-comercial, familia y laboral, para las que el Estado colombiano no brinda la información respectiva.

El informe de la Coalición no se detiene en este aspecto, y se destaca que sus críticas se centran más en las barreras que afrontan las personas con diversidad funcional, en tanto son procesadas, pero poco en su rol de víctimas y los mecanismos para ejercer plenamente sus derechos en tanto tales.

El Comité, por su parte, sí hace un llamado al Estado colombiano para que "Adopte los ajustes de procedimiento judiciales que garanticen el desempeño de personas con discapacidad en cualquiera de las funciones y etapas de los procesos", que claramente constituyen el cumplimiento del deber de adoptar disposiciones de Derecho interno y no se limitan al ámbito exclusivamente criminal.
El Estado Parte también informa que el Instituto Nacional de Medicina Legal y Ciencias Forenses (INMLyCF) cuenta con una funcionaria conocedora del lenguaje de señas y que en los encuentros nacionales se han realizado campañas de sensibilización. $\mathrm{Al}$ respecto es importante señalar que, por la fecha de presentación del informe inicial, la evaluación del Comité y la participación de la Sociedad Civil ya han transcurrido varios años y actualmente no sólo el INMLyCF, sino cualquier entidad puede prestar sus servicios a la población usuaria de lengua de señas colombiana con el apoyo del "Centro de Relevo" . Convendría indagar con el Consejo Superior de la Judicatura qué tipo de articulación existe en dicho sector para acercar al personal judicial a la población sorda. No obstante, es claro que el informe del país no da cuenta de la garantía de la comunicación a otras personas con diversidades funcionales. La Coalición no se pronunció al respecto en su informe alternativo, pero el Comité aborda esta preocupación en las recomendaciones contenidas en el literal C.

Finalmente, el informe de País (presentado por el Estado colombiano al Comité) aborda muy superficialmente el ámbito territorial, destacando la necesidad de sensibilización y formación de todo el personal de la rama judicial y reconoce problemas de accesibilidad. La Coalición tampoco se pronuncia sobre el impacto territorial y el Comité aborda desde una perspectiva nacional (que implica las dimensiones local y regional) tales obligaciones en las recomendaciones del literal c) y d). En este punto es importante destacar cómo los 3 documentos desconocen otras formas de acceso a la justicia, de especial relevancia y connotación territorial como la justicia comunitaria, los mecanismos de resolución pacífica de los conflictos como la conciliación, la amigable composición, entre otras, y la jurisdicción especial indígena, información que no está disponible por parte de las autoridades nacionales. Estos retos hacen pertinente el llamado de Aimee Mullins, en el sentido de que

I. http://www.centroderelevo.gov.co/632/w3-channel.html. 
las personas con diversidad funcional deben prepararse para la adversidad (Mullins, 2009) y el Estado debe garantizar así para hacer efectivo el goce de sus derechos.

Teniendo en cuenta que según las estadísticas, 322 millones de personas viven con depresión, es decir, que un $4,4 \%$ de la población del planeta tiene depresión (OMS, 20I7) un solo episodio no alude a discapacidad, ni siquiera en el caso de presentar varios episodios de psicosis o de manía. En el caso de esquizofrenia o del trastorno bipolar tampoco hace discapacitado al que los padece: los incapacita temporalmente, pues entre los episodios sintomáticos, hay periodos libres de síntomas, en los que ellos pueden asumir su rol social, familiar y laboral sin mayores contratiempos. Dicho en otras palabras, asegurar que una persona con el solo diagnóstico médico de depresión, manía, ansiedad o psicosis es una persona con discapacidad psicosocial, es casi como afirmar que todos los usuarios de lentes correctivos presentan discapacidad visual. Expuesto este punto se colige que las demandas de la convención sólo aplicarían a las personas que verdaderamente presentan discapacidad psicosocial, al menos acorde con la definición de la Convención (Organización de Naciones Unidas, 2007). Por lo que se puede concluir que tanto el informe oficial como el informe alternativo tendrían un grave error de fondo.

En el caso de las medidas de seguridad para las personas con enfermedad mental declaradas inimputables, hay que advertir que la escuela penal en Colombia es de corte positivista en donde tiene en cuenta al delito y la persona que cometió el delito, de tal forma que si aquel a quien se le imputa una conducta punible pero tiene alteradas su capacidad de comprensión y de autodeterminación no recibe un pena sino una medida de seguridad que es una restricción del derecho a la libertad como consecuencia de la comisión de un hecho punible y es considerada en otros países como una sanción alternativa, sin embargo es cierto que la modalidad de la medida (internación hospitalaria, en casa o libertad vigilada) y su duración depende de muchos factores, pero es el juez quién en última instancia decide aceptar o no las recomendaciones de los actores involucrados en el proceso (Instituto Nacional de Medicina Legal y Ciencias Forenses, 20I0). Ahora, si se va al extremo, las personas con un diagnóstico de enfermedad mental no reciben ningún tipo de restricción, contravención o castigo por haber cometido un delito, cualquier individuo con un diagnóstico llámese depresión o ansiedad podría matar, violar, torturar y/o agredir sin recibir una pena. Y casos muy publicitados en los medios han intentado evadir su responsabilidad de esta forma.

Con relación a la mencionada guía sobre medidas de seguridad del Instituto Nacional de Medicina Legal y Ciencias Forenses, es importante precisar que este instituto es una entidad adscrita a la Fiscalía General de la Nación que funge como auxiliar de la justicia y no cumple funciones de legislador (Instituto Nacional de Medicina Legal y Ciencias Forenses, 2004), por lo que la guía solo podría ser modificada cuando cambie el código penal y el código de procedimiento penal.

Dos aseveraciones en el informe alternativo son de importancia capital, la primera que asegura que las personas con discapacidad psicosocial reciben tratamientos forzados que incluyen la terapia electroconvulsiva, sin embargo, no hay máquinas de ese tipo en la red pública del distrito capital y en Cundinamarca (quienes son las que atienden la mayoría de personas con enfermedades mentales). Es recomendable antes de enviar un informe de estas características, pedir al menos en Bogotá, las estadísticas reales de esta práctica, pena de lo expresado en el informe sea desvirtuado públicamente. La segunda aseveración es la llamada sobremedicación, concepto que no se explica, solo se deja entrever como un comentario.

Las internaciones permanentes son de dos tipos: aquellas de origen judicial (ya comentada) y de origen no judicial, esta última merece algunos comentarios: con relación a las personas con discapacidad psicosocial verdadera, si bien este proceso se inicia con la solicitud del representante legal, solamente el juez puede autorizarlo, pero no se hace solamente en 
términos de peligrosidad, aunque es el factor más frecuente. En los casos de adultos con discapacidad intelectual leve en medida de protección del ICBF permanecen recluidos perennemente en instituciones sin posibilidad egreso. No hay sistema intermedio en la que haya salidas sin acompañante, una alternativa podría ser el programa de aldeas infantiles donde una persona se haga cargo de ellos, tengan una familia y reciban apoyo académico y laboral hasta lograr su independencia. En ambos casos, se viola el artículo i 2 de la Convención.

Por otro lado, es necesario tener en cuenta el artículo 2I para facilitar a todas las personas con discapacidad el acceso a la información en modos, medios y formatos de comunicación accesibles. Teniendo en cuenta lo anterior, se debe considerar que el lenguaje es un elemento histórico social que está fuera del sujeto y muchas veces se ha promulgado sin conciencia debido a que está objetivado con determinados cargos semánticos. Lo anterior sucede porque el lenguaje en sí mismo es un conjunto de objetivaciones comunes a un grupo de sujetos y su disponibilidad y durabilidad les permite una trascendencia en el tiempo.

El conocimiento de la vida cotidiana se estructura en términos de relevancias, percepciones, intereses y análisis pragmáticos en diferentes contextos comunicativos, ya que la comunicación no es un acto individual sino colectivo y para grandes masas. Por lo tanto, es importante tener en cuenta la red simbólica del lenguaje, donde existen una pluralidad de símbolos, los cuales dependen uno del otro y están siempre dispuestos a vincular nuevos símbolos; confiriendo diferentes significados según la composición y el tipo de símbolo y las relaciones que hallan entre sí (Berger y Luckmann, 200I: 5I). Por lo anterior, es importante cambiar la terminología y usarla de forma adecuada, creando herramientas que reglamenten el uso del lenguaje en torno a la replicación de información por medios masivos de la comunicación, permitiendo la transformación de la naturaleza de los conceptos alrededor de la discapacidad.
En lo referente al artículo I 5, la Convención manifiesta que es necesaria la creación de un protocolo o mecanismo nacional que prevenga la tortura y supervise las instituciones de reclusión, asimismo, considera pertinente crear legislación en el área. Sin embargo, es evidente que aún no existen acciones en contra de estas prácticas de violencia como es manifestado en el informe alternativo, adicionalmente no se cuenta con registro estadístico de dichos eventos y se evidencia que las poblaciones más vulnerables son más propensas a eventos de este tipo, incluso en los servicios de salud.

Según el Informe sobre la tortura y otros tratos o penas crueles, inhumanos o degradantes (ONU, 20I3), el principal escenario donde se evidencian malos tratos y tortura, es el de atención en salud dado por el "trato inhumano y degradante"; lo cual debe obligar a los estados a tomar acciones que eviten estas posibles violaciones y que garanticen condiciones adecuadas para la atención en salud, emanando los derechos de libertad y dignidad de las personas.

\section{Conclusiones}

La mirada holística de Martha Nussbaum en su llamado enfoque de capacidades nos presenta una nueva perspectiva sobre las personas con capacidades diversas que va desde el valor intrínseco del ser humano solo por el hecho de ser humano y devuelve a todas las personas con o sin discapacidad el reconocimiento de su valía más allá del concepto de corporalidad normativa y cogniciones diversas (Rodríguez, s.f.). Aimee Mullins en su vídeo nos enseña cómo el enfocarse en sus propias capacidades y no en sus falencias, la motiva todos los días a vivir una vida buena (Mullins, 2009). El informe inicial del Estado colombiano, el informe alterno de la Coalición y las observaciones finales del Comité evidencian que Colombia aún tiene un largo camino que recorrer para cumplir lo exigido y realizar los cambios pertinentes. 
Berger, P. y Luckmann, T. (200I): La construcción social de la realidad, Buenos Aires: Amorrortu.

Comité sobre los Derechos de las Personas con Discapacidad (2016a): Informe alternativo de la Coalición colombiana para la implementación de la Convención sobre los Derechos de las Personas con Discapacidad (en línea). <http://discapacidadcolombia. com/phocadownloadpap/PUBLICACIONES_ ARTICULOS/Informe $\%$ 2oAlterno $\% 20$ Coalicion \% 20Colombiana \% 20-\% 2oesp.pdf>, acceso I 8 de diciembre de 2017.

Comité sobre los Derechos de las Personas con Discapacidad (2016b): Observaciones finales sobre el informe inicial de Colombia. CRPD/C/ $\mathrm{COL} / \mathrm{CO} / \mathrm{I}$ (en línea). <http://tbinternet. ohchr.org/_layouts/treatybodyexternal/ Download.aspx?symbolno=CRPD/C/COL/ $\mathrm{CO} / \mathrm{I} \& \mathrm{Lang}=\mathrm{Sp}>$, acceso I4 de abril $20 \mathrm{I} 7$.

Instituto Nacional de Medicina Legal y Ciencias Forenses (2004): Objetivos y funciones (en línea). < http://www.medicinalegal.gov.co/ objetivos-y-funciones;jsessionid=oD $99{ }_{9}{ }_{23} 3$ Do F82AAA6A82>, acceso I 8 de abril de 2017.

Instituto Nacional de Medicina Legal y Ciencias Forenses (2010): Guía para la realización de pericias psiquiátricas forenses sobre mantenimiento, cambio o levantamiento de medidas de seguridad en inimputables (en línea). <http://www.medicinalegal.gov.co/ documents/48758/7808I/G3.pdf/a65a2ad2$\mathrm{f}_{3}$ ef-4873-98a9-46I 5 f 9 eo 5 obb>, acceso I 8 de abril de 2017 .

Mullins, A. (2009): The opportunity of adversity [Archivo de vídeo] (en línea). <https://www.ted. com/talks/aimee_mullins_the_opportunity_of_ adversity\#t-49656>, acceso I9 de marzo 2017.

Organización de Naciones Unidas, ONU (2007): Convención sobre los derechos de las personas con discapacidad (en línea). <http://www. un.org/esa/socdev/enable/documents/tccconvs. pdf $>$, acceso I 9 de abril de 2017.

Organización de las Naciones Unidas, ONU (20I3): Informe del Relator Especial sobre la tortura y otros tratos o penas crueles, inhumanos o degradantes, Juan E. Méndez. Consejo de Derechos Humanos (en línea). <http://www.ohchr.org/Documents/HRBodies/ HRCouncil/RegularSession/Session22/AHRC-22-53_sp.pdf>, acceso I9 de abril de 2017.

Organización Mundial de la Salud (20I7): Depresión. Nota descriptiva (en línea). <http:// www.who.int/mediacentre/factsheets/fs36 6 /es/>, acceso I 8 de diciembre de 20 I 7.

República de Colombia (2013): Informe inicial sobre la implementación de la Convención sobre los Derechos de las PcD en Colombia (en línea). <https://discapacidadcolombia. com/phocadownloadpap/PUBLICACIONES_ ARTICULOS/Informe $\%$ 2oEstado $\% 20$ Colombiano \% 2oImplementacion \% 20CDPD. pdf $>$, acceso I4 de abril 2017.

Rodríguez, R. (s.f.). Martha Nussbaum: Las capacidades humanas y la vida buena (en línea). <http://www.ieturolenses.org/revista_ turia/index.php/actualidad_turia/marthanussbaum-las-capacidades-humanas-y-la-vidabuena>, acceso I 2 de marzo de 2017. 